\title{
Reflets
}

Revue d'intervention sociale et communautaire

\section{Profilage social et judiciarisation des personnes itinérantes à Montréal - Opération Droits Devant et autres consolations!}

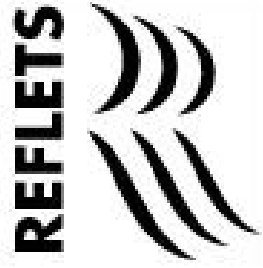

\section{Bernard St-Jacques}

Volume 22, numéro 1, printemps 2016

URI : https://id.erudit.org/iderudit/1037167ar

DOI : https://doi.org/10.7202/1037167ar

Aller au sommaire du numéro

Éditeur(s)

Reflets, Revue d'intervention sociale et communautaire

ISSN

1203-4576 (imprimé)

1712-8498 (numérique)

Découvrir la revue

Citer cet article

St-Jacques, B. (2016). Profilage social et judiciarisation des personnes itinérantes à Montréal - Opération Droits Devant et autres consolations! Reflets, 22(1), 173-182. https://doi.org/10.7202/1037167ar d'utilisation que vous pouvez consulter en ligne. 


\section{Profilage social et judiciarisation des personnes itinérantes à Montréal - Opération Droits Devant et autres consolations!}

Bernard St-Jacques, organisateur communautaire

Réseau d'aide aux personnes seules et itinérantes de Montréal (RAPSIM)

À partir de la fin des années 1990, les organismes communautaires en itinérance à Montréal ont constaté une recrudescence d'une certaine forme de criminalisation de la pauvreté basée sur la remise plus systématique de contraventions par les policiers aux sansabri de la métropole. Dès lors, les intervenantes et intervenants du milieu se sont mis à accompagner des personnes à la cour municipale comme ils le faisaient au bureau de l'aide sociale et tentaient, par le fait même, de colliger les contraventions reçues, question d'en analyser les infractions reprochées. Même si l'on n'utilisait pas encore le vocable de profilage social, un caractère discriminatoire dans les motifs invoqués faisait déjà partie des principaux constats de l'époque.

La problématique de la judiciarisation des personnes en situation et à risque d'itinérance est en partie attribuable à l'effet de mode de la revitalisation des centres des grandes villes nordaméricaines. D'abord, une pression est exercée afin de rendre plus attirants ces lieux pour le tourisme, l'industrie culturelle et l'offre de condominiums pour les personnes en mesure de se les payer, pression qui se trouve transposée sur la population marginalisée qui y est présente depuis toujours. S'ajoute à cela l'évolution des pratiques sécuritaires, un enjeu qui viendra prendre 
une place impressionnante avec la mise en place de la police de quartier à Montréal et le ciblage de populations jugées comme étant à risque de commettre de petits délits. Enfin, la fermeture de l'espace public, perceptible au début des années 2000, est un phénomène qui passe par le changement de statut de plusieurs places et squares transformés en parcs, permettant ainsi le recours à une nouvelle réglementation municipale destinée à ces lieux spécifiques (fermeture des parcs la nuit, utilisation du mobilier urbain, etc.) et une augmentation de la présence policière. Les tensions déjà présentes dans la rue atteindront leur paroxysme en 2004, année où il s'est donné le plus de contraventions dans les espaces publics à Montréal en même temps que s'amorçait la riposte des organismes du milieu. Il se dégageait un sentiment d'injustice chez les personnes fréquentant la rue, tout comme un sentiment d'impuissance chez les intervenantes et intervenants leur venant en aide. Ces sentiments accompagnaient le quotidien de plusieurs personnes se déplaçant d'un quartier à l'autre pour fuir la répression ou séjournant en prison pour le non-paiement de leurs amendes. Depuis, bien de l'eau a coulé sous les ponts, non sans batailles ni sensibilisation.

Le présent article vise à dépeindre l'évolution des pratiques rattachées au phénomène de profilage social à Montréal et le déploiement d'une pratique de défense des droits pour y faire face, l'Opération Droits Devant. Pour ce faire, nous allons d'abord étayer certains éléments venant soutenir l'existence du phénomène de même que sa reconnaissance et présenter des réponses apportées par les autorités en place. Par la suite, nous nous concentrerons sur les origines et les actions découlant de l'Opération Droits Devant. Enfin, nous dresserons un état de la situation actuelle en intégrant les enjeux liés aux différents projets de réaménagement urbain et les défis de la cohabitation sociale.

\section{Démonstration du phénomène}

«Ce n'est pas faute d'avoir essayé ». Combien de fois avons-nous employé cette expression à la suite de nombreuses tentatives 
pour démontrer le caractère discriminatoire dans la remise de contraventions aux personnes en situation d'itinérance? Certes, on touchait une corde sensible à laVille de Montréal et au Service de police de la Ville de Montréal (SPVM) en affirmant qu'ils s'adonnaient à un traitement répréhensible et "anti-pauvres". Encore aujourd'hui, le phénomène est quelque peu reconnu, mais attribué à une très faible proportion des effectifs policiers, et cette reconnaissance s'est avérée moins perceptible dans les aveux formulés que dans les gestes posés de manière très progressive. En dehors des efforts déployés par le milieu communautaire pour dénoncer ce profilage social, deux moments forts de la démonstration de son existence ont contribué à le faire connaitre et reconnaître.

Tout d'abord, en 2005, la chercheure Céline Bellot et son équipe ont réalisé une première étude d'envergure destinée à témoigner des effets de la judiciarisation chez les personnes itinérantes et à en faire la démonstration chiffrée à même les données de la cour municipale. Cette première de trois études réalisées à Montréal a créé une véritable onde de choc dans le public tout en venant confirmer bien des craintes et constats formulés dans le milieu de l'itinérance (Bellot, et collab., 2005). L'étude se déclinait en une analyse de quelque 22685 contraventions remises à 4036 personnes entre 1994 et 2004. En fin de compte, on a constaté qu'il s'en est donné 7 fois plus en 2004 (la pire année) que dix ans plus tôt, et on a estimé que dans $72,3 \%$ des cas où une contravention a été remise, les personnes les payaient par un séjour en prison. En outre, on a pu confirmer l'existence de situations de surjudiciarisation dans lesquelles des personnes avaient reçu de nombreuses contraventions en même temps ou dans un court laps de temps. Ainsi, $60 \%$ de l'ensemble des contraventions avaient été dispensés à 600 personnes ayant reçu plus de 10 contraventions chacune, soit moins de $15 \%$ de l'échantillon total de 4036 personnes. Enfin, l'étude venait révéler l'emballement et l'explosion des coûts pour les personnes judiciarisées et pour le système pénal. On observera par la suite une baisse du nombre de contraventions remises dans l'espace public. Bref, cette première recherche a permis d'offrir une importante 
crédibilité à la dénonciation du phénomène du profilage social tout en constituant un levier pour la transformation des pratiques policières à venir.

Ensuite, un autre appui de taille est venu de la Commission des droits de la personne et des droits de la jeunesse du Québec (CDPDJQ). En effet, un avis produit en 2009 ne constituait ni plus ni moins qu'un accablant rapport de plus de 200 pages, une analyse sociale et juridique sur le profilage des sans-abri à Montréal (CDPDJQ, 2009). Son contenu touchait autant les directives policières et le pouvoir discrétionnaire des policiers que l'application de la réglementation et le recours possible à la prison pour non-paiement d'amendes. Non seulement on y alléguait que la Ville de Montréal et le SPVM s'adonnaient de façon systémique à des pratiques de profilage social, mais les solides recommandations du document concernaient tous les niveaux du processus de judiciarisation : réglementation, répression, prison, etc. De plus, on y soutenait la nécessité d'élaborer des mesures sociales permettant de rendre caduc le recours à la contraventionnalisation, à commencer par la mise en place d'une Politique nationale de lutte à l'itinérance qui sera présentée en 2014.

Les autorités municipales reconnaîtront l'existence de pratiques de profilage social, sans parler ouvertement de son caractère systémique. Qui plus est, quelques gestes posés auront nécessairement eu certains effets. Tout d'abord, au niveau du discours, on a pu sentir un adoucissement du ton pris par les autorités quand elles abordaient la question du partage de l'espace public. Il en a été de même des plans d'action et autres documents de laVille de Montréal et du SPVM qui jadis ciblaient davantage des populations marginalisées en leur accolant des étiquettes et en laissant entendre qu'elles étaient davantage susceptibles de commettre des incivilités. De plus, la baisse relative et progressive du nombre de contraventions remises dans l'espace public, notamment certaines liées aux infractions les plus farfelues (par exemple jeter sa cendre de cigarette ou du liquide par terre), était due à de nouvelles directives émises aux patrouilleurs. $\mathrm{Si}$, au départ, la Ville n'admettait pas en tant que tel le profilage, force lui était de constater qu'effectivement, la judiciarisation était une 
pratique courante et dommageable. L'un de ses premiers gestes a donc été de mettre à contribution sa cour municipale dans le développement de programmes spéciaux, notamment avec le soutien de procureurs et de percepteurs désignés, pour sortir le plus grand nombre de personnes du système judiciaire. Finalement, un moratoire sur l'émission des mandats d'emprisonnement pour non-paiement d'amendes est toujours en vigueur aujourd'hui.

Le vocable profilage social a finalement été employé, quoique bien après 2005. En 2011, lors d'une refonte de la Charte montréalaise des droits et responsabilités, la Ville décidait d'y ajouter les profilages racial et social aux motifs de discrimination potentielle. Plus encore, le SPVM et laVille publiaient, en 2012, le Plan stratégique en matière de profilage racial et social, destiné à définir le phénomène et à donner une formation à son personnel sur le phénomène, à tenter un rapprochement avec les personnes qui en sont victimes et à développer des mécanismes visant à sanctionner les comportements répréhensibles en la matière.

En somme, de telles démonstrations et une ouverture à admettre l'existence du profilage social ont contribué à son atténuation. Mais derrière ces moments forts et l'ouverture manifestée, il y a eu tout un mouvement social et communautaire qui s'est mis en branle à partir de 2003.

\section{L'Opération Droits Devant}

L'Opération Droits Devant est une pratique de défense de droits qui avait comme premier objectif de mettre en commun les actions déjà réalisées par les intervenantes et intervenants du milieu. Néanmoins, à la nécessité de faire connaitre les rudiments du système judiciaire pour venir en aide aux personnes et documenter la situation, en particulier à partir de l'information inscrite sur les contraventions reçues, s'ajoutait l'urgence d'atteindre l'ensemble de la population pour l'interpeller sur cette problématique inacceptable et suggérer des solutions de rechange. Ainsi, l'Opération Droits Devant (ODD) est dévoilée en mai 2003 par le Réseau d'aide aux personnes seules et itinérantes de Montréal 
(RAPSIM) et la Table de concertation jeunesse-itinérance du centre-ville. Elle consiste en une pratique de défense des droits dans laquelle se mobilisent les actrices et acteurs du milieu en vue de documenter et de dénoncer la situation de judiciarisation et de sensibiliser la population ainsi que les actrices et acteurs juridiques et politiques. Elle disposait aussi d'un volet plus individuel constitué d'outils permettant aux intervenantes et intervenants de démystifier le complexe système judiciaire.

Dès ses débuts, l'ODD constituait un lieu de discussion et de mobilisation bien fréquenté par les intervenantes et intervenants, tant pour prendre le pouls de la situation sur le terrain que pour se vider le cœur concernant la difficile réalité vécue par les personnes judiciarisées. À la fin de l'année 2003, les groupes sortaient en conférence de presse avec le chanteur Dan Bigras, porte-parole du Refuge des jeunes de Montréal, dévoilant les premiers constats tirés des contraventions colligées par les organismes. En 2004, l'ODD se fera connaitre grâce à une première activité de sensibilisation réalisée dans les rues de Montréal et consistant, pour les intervenantes et intervenants et les personnes judiciarisées, à distribuer des contraventions symboliques à monsieur et madame Tout-le-Monde.

C'est dans cette même période que le RAPSIM s'est adressé pour la première fois à la Commission des droits de la personne et de la jeunesse du Québec relativement à la question de la judiciarisation des personnes itinérantes en lui demandant de se prononcer sur le potentiel discriminatoire dans la remise des contraventions à ces personnes. D'abord davantage encline à tenter de trouver des solutions avec l'ensemble des actrices et acteurs interpellés, la Commission a fini par déposer près de six ans plus tard, en novembre 2009, l'impressionnant rapport dont nous avons parlé plus tôt. L'Opération Droits Devant a exercé des pressions sur la Commission et l'a alimentée sur la question pendant toutes ces années pour en faire une institution de premier plan en appui à la lutte contre la judiciarisation et le profilage social. Outre le milieu de la recherche et la Commission, l'ODD s'est adjoint le concours et l'appui de nombreuses organisations, notamment dans le domaine juridique, où des avocates et avocats, des groupes 
d'étudiantes et étudiants, des groupes de défense de droits et le Barreau du Québec ont été mis à contribution.

L'Opération Droits Devant a tenu d'autres activités de mobilisation, dont des rassemblements aux abords de l'hôtel de ville, de la cour municipale et de l'arrondissement Ville-Marie. On y dénonçait notamment l'emprisonnement pour non-paiement d'amendes ou encore le fait d'appliquer certains règlements municipaux ou d'en étendre l'utilisation. Une quinzaine de forums ont aussi été organisés, dont une majorité visait la participation et la formation des membres du RAPSIM et la discussion entre ces derniers sur l'enjeu de la judiciarisation. Étant au cœur des préoccupations, un nombre grandissant de personnes judiciarisées ont été invitées au fil des années à venir témoigner de leur situation.

Par ailleurs, le RAPSIM constatait que la défense collective des droits donnait davantage de résultats et que la formation des intervenantes et des intervenants du milieu, déjà surchargés, ne leur permettait pas de régler autant de situations judiciaires qu'ils ne l'auraient souhaité. C'est pourquoi, à la fin de l'année 2006, le RAPSIM a créé sous sa bannière la Clinique Droits Devant. Véritable service d'accompagnement dans l'arène judiciaire, cette clinique, portée par des intervenantes et intervenants sociaux, permet regarder des dossiers de contraventions des personnes et de voir avec elles les possibilités de règlement : ententes sur des travaux compensatoires ou sur des paiements, contestation et présence à la cour, intégration aux programmes spéciaux à la cour. Par exemple, il y a le Programme d'accompagnement justice-itinérance à la cour (PAJIC), qui permet à la personne de voir nombre de ses contraventions radiées du système, après avoir démontré l'amélioration de sa situation globale en matière de logement, de consommation, de réinsertion et d'emploi, etc. À cela s'ajoutent des formations dispensées aux intervenantes et intervenants et des ateliers sur les droits offerts aux personnes judiciarisées. La Clinique Droits Devant est d'ailleurs devenue un organisme autonome en 2014. 
Une douzaine d'années après sa création, l'Opération Droits Devant doit continuer de faire la démonstration de la persistance du profilage social et de ses effets. Bien que des progrès soient à souligner et que l'on assiste à une baisse assez significative du nombre de contraventions, on ne peut pas dire que les heurts, les contraventions, les abus et le profilage sont devenus inexistants dans l'espace public aujourd'hui. Des portraits encore récents de la situation et des perceptions des intervenantes et intervenants à l'égard du profilage font ressortir de timides améliorations, notamment en ce qui a trait au harcèlement, aux abus policiers physiques et verbaux (RAPSIM, 2014). Alors qu'on se serait imaginé être plus loin dans la défense des droits et permettre aux personnes judiciarisées de réintégrer une citoyenneté perdue, force est de constater qu'on est encore à travailler pour faire face à leur sentiment d'injustice et régler leur situation judiciaire individuelle. Néanmoins, la situation peut encore s'améliorer.

\section{Après les contraventions et le profilage, la cohabitation?}

La judiciarisation de personnes itinérantes et, plus largement, le profilage social constituent le révélateur et les effets des ratés du partage de l'espace public et de la cohabitation sociale. On peut tenter de séparer la situation des 15 dernières années en deux périodes dans lesquelles on peut situer le profilage et la cohabitation, espérant ainsi en être rendu au tout début d'une troisième phase. La première période s'échelonnant à peu près de la fin des années 1990 à 2005 en constitue une qui est fondée sur les contraventions et où la répression comme l'expulsion deviennent caractéristiques de l'approche adoptée pour aborder la cohabitation. Plus graduellement par la suite, on en arrivera à un nouveau paradigme, celui du profilage social, dans lequel on observe d'autres de ses aspects et pratiques, les contraventions étant en baisse. En ce qui a trait à la cohabitation, on assistera au développement de projets de réaménagement et de réappropriation d'espaces publics par les citoyennes et les citoyens, projets dans 
lesquels on se dit davantage ouvert à partager ces lieux avec les personnes marginalisées. Toutefois, on n'en est certes pas rendu à une véritable inclusion, celle-ci étant absolument conditionnelle à la participation aux activités déterminées par la majorité et à un respect démesuré des lieux transformés.

Dès lors, peut-on espérer en être aux tout premiers balbutiements d'une troisième et nouvelle phase, qui s'inscrirait dans le contexte de la publication récente de la Politique nationale de lutte à l'itinérance (2014)? Selon celle-ci, l'inclusion deviendrait-elle la caractéristique principale tant des moyens déployés (contre les contraventions et le profilage) que de la situation générale (contre la répression et la cohabitation conditionnelle)? Dans ce cas, le profilage social ne serait pas encore totalement évacué, mais on pourrait démontrer que des efforts vont véritablement en ce sens. Qui plus est, le mantra ne serait plus de favoriser une inclusion conditionnelle à l'utilisation de l'espace public, mais bien d'offrir des activités qui répondent aux besoins des personnes en situation d'itinérance, d'autres qui répondent aux besoins du reste de la population et d'autres encore qui répondent aux besoins des deux. Le tout doit s'appuyer sur des mécanismes permettant de prendre en compte le point de vue des personnes qui n'ont pas voix au chapitre. C'est ce à quoi laVille de Montréal et le SPVM affirment nous convier avec beaucoup de candeur, respectivement dans le récent Plan d'action montréalais en itinérance 2014-2017 (Ville de Montréal, 2014) et dans le nouveau Plan stratégique en matière de profilage racial et social (SPVM, 2012) actuellement mis en place. Jamais n'aura-t-on vu autant de lieux et d'espaces publics aussi significatifs pour les personnes en situation d'itinérance être appelés à changer de vocation, comme le Square Cabot, le Parc Émilie-Gamelin et, plus encore, le Carré Viger. En même temps, jamais n'aura-t-on vu autant d'ouverture à en faire des lieux véritablement inclusifs. Dès lors, peut-on espérer prendre la balle au bond et en profiter, par le fait même, pour rendre caduc le profilage social? Voilà encore une utopie qui ne demande pas mieux que de devenir réalité. À suivre! 


\section{Bibliographie}

BELLOT, Céline, et collab. (2005). Judiciarisation et criminalisation des populations itinérantes à Montréal, Montréal, Université de Montréal, 144 p.

COMMISSION DES DROITS DE LA PERSONNE ET DES DROITS DE LA JEUNESSE DU QUÉBEC (2009). La judiciarisation des personnes itinérantes à Montréal : un profilage social, Montréal, Commission des droits de la personne et des droits de la jeunesse, $202 \mathrm{p}$.

GOUVERNEMENT DU QUÉBEC (2014). Ensemble, pour éviter la rue et en sortir. Politique nationale de lutte à l'itinérance, Québec, gouvernement du Québec, réf. du 9 janvier 2016, http://publications.msss.gouv.qc.ca/msss/fichiers/2013/13-846-03f.pdf

RÉSEAU D'AIDE AUX PERSONNES SEULES ET ITINÉRANTES DE MONTRÉAL (2014). Profilage social et judiciarisation : portrait des recours, des abus policiers et autres agents en situation d'autorité, Montréal, RAPSIM, 38 p.

SERVICE DE POLICE DE LA VILLE DE MONTRÉAL (2012). Plan stratégique en matière de profilage racial et social, Montréal, SPVM, $46 \mathrm{p}$.

VILLE DE MONTRÉAL (2014). Plan d'action montréalais en itinérance 2014-2017. Parce que la rue est une impasse, Montréal,Ville de Montréal, 19 p.

VILLE DE MONTRÉAL (2011). Charte montréalaise des droits et responsabilités (2e éd.), Montréal, Ville de Montréal, 28 p.

Ces documents sont disponibles au www.rapsim.org. 